\title{
Sistema de Pastejo Rotativo "Ponta e Rapador" para Novilhas de Corte. 1. Desenvolvimento Corporal ${ }^{1}$
}

\author{
Octaviano Alves Pereira Neto², José Fernando Piva Lobato ${ }^{3}$, Álvaro Simeone ${ }^{4}$
}

\begin{abstract}
RESUMO - O objetivo deste trabalho foi avaliar o desenvolvimento de novilhas de corte no sistema de pastejo rotativo "ponta" e "rapador" durante o primeiro (Ano-1) e segundo (Ano-2) invernos/primaveras pós-desmama até o final do primeiro acasalamento aos 22-24 meses de idade. Setenta e seis bezerras de corte mestiças foram mantidas durante Ano-1 e Ano-2 em pastagem nativa melhorada de azevém (Lolium multiflorum L.) e trevo vesiculoso Yuchi (Trifolium vesiculosum cv. Yuchi). No período de verão/outono e acasalamento os animais foram mantidos em campo natural, em lote único. Os tratamentos Ponta (TP): grupo consumindo a metade superior da pastagem disponível; Rapador (TR): consumindo a metade inferior da pastagem foram testados. A evolução do peso vivo (PV) em cada etapa do experimento e ganho de peso médio diário (GMD) por período foram analisados. O peso vivo de TP diferiu do TR ao final do Ano-1 (195,6 vs. 168,1 kg), ao final do Ano-2 (316,3 vs. 274,1 kg) e ao início (318,5 vs. 275,7 kg) e final (341,5 vs. 304,4 kg) do acasalamento, respectivamente. O maior PV foi atribuído ao maior GMD obtido por TP sobre TR durante o uso da pastagem melhorada, com GMD de 0,790 vs $0,563 \mathrm{~kg} / \mathrm{dia}$ e 0,753 vs. 0,522 kg/dia, no Ano-1 e Ano-2, respectivamente. Houve inversão no GMD com melhor desempenho do TR em relação ao TP $(0,377$ vs. $0,303 \mathrm{~kg} / \mathrm{dia})$ no acasalamento. O sistema de recria de novilhas para reposição, favorecendo determinado grupo de animais (TP), é uma das alternativas para obter pesos vivos satisfatórios, tornando-as aptas à concepção aos 22-24 meses de idade.
\end{abstract}

Palavras-chave: gado de corte, novilhas de reposição, pastagem melhorada, pastejo rotativo, sistema de utilização de pastagens

\section{Rotational Grazing System "First and Last Grazers" for Beef Heifers. 1. Body Development}

\begin{abstract}
The objective of this work was to evaluate the development of beef heifers in a rotational grazing system as "first grazers" and "last grazers" during the first (Year-1) and second (Year-2) winter/spring seasons post weaning until the end of the first mating at 22-24 months of age. Seventy-six crossbred female calves were maintained during Year-1 and Year-2 on improved natural pasture of ryegrass (Lolium multiflorum L.) and arrowleaf clover (Trifolium vesiculosum cv. Yuchi). During the summer/autumn period and mating season, the heifers were maintained on natural pasture as a unique mob. The First grazers (FG) treatments: group eating the superior half of the available pasture; Last grazers (LG): group eating the inferior half of the available pasture, were tested: The live weight evolution (LW) in each phase of the experiment and average daily weight gain (ADG) per period were analyzed. The live weight of FG differed from LG at the end of the Year-1 (195.6 vs. $168.1 \mathrm{~kg})$, at the end of the Year-2 (316.3 vs. $274.1 \mathrm{~kg})$, at the beginning (318.5 vs. 275.7 $\mathrm{kg}$ ) and at the end (341.5 vs. $304.4 \mathrm{~kg}$ ) of the mating period, respectively. The higher LW was due to the higher ADG obtained by FG over LG during the use of the improved pastures, with ADG of .790 vs. $.563 \mathrm{~kg} /$ day and $.753 \mathrm{vs.} \mathrm{.522} \mathrm{kg/day,} \mathrm{at} \mathrm{Year-1} \mathrm{and} \mathrm{Year-2,}$ respectively. There was an inversion in the ADG with a better performance of LG in relation to FG (.377 vs. .303 kg/day, respectively), in the mating period. The raising systems for replacement heifers that favored a determined animal group (FG) is one of the alternatives to obtain a satisfactory live weights, making them able to conceive at 22-24 months of age.
\end{abstract}

Key Words: beef cattle, replacement heifers, improved pasture, rotational grazing system, pasture utilization system

\section{Introdução}

É normal o fato de os rebanhos de cria bovina produzirem suas próprias novilhas de reposição. Assim, é importante estabelecer programas eficientes de recria e seleção para a obtenção do maior número possível de novilhas aptas à reprodução na idade e época desejada (BOLZE e CORAH, 1993; PEREIRA e LOBATO, 1998). Segundo PARSONS e ALLISON (1991), para a análise de eficiência de sistemas de cria, o principal indicador é o máximo desempenho reprodutivo das fêmeas e nos de engorda, a produtividade de carne por animal ou por área. Portanto, essas são as metas de trabalho do produtor

\footnotetext{
${ }^{1}$ Parte da Tese de Mestrado do primeiro autor.

2 Médico Veterinário (UFPel) e Mestre em Zootecnia (UFRGS).

${ }^{3}$ Professor Adjunto IV, Departamento de Zootecnia, Faculdade de Agronomia, UFRGS. Bolsista CNPq - 1A, Caixa Postal, 776 - $90.001-970$ - Porto Alegre, RS.

${ }^{4}$ Professor, Cátedra de Bovinotecnia, Faculdade de Agronomia, Universidade de la República, Paysandú, Uruguai.
} 
e dos técnicos que lhes dão suporte, visando maximizar os resultados do sistema de produção proposto.

A redução da idade ao primeiro acasalamento para os dois anos de idade ou menos resulta em impactos econômicos mais significativos sobre a produtividade do setor pecuário do que a redução da idade de abate de novilhos dos quatro para os dois anos de idade (GRAWUNDER e MIELITZ, 1979).

LAMOND (1970) definiu como "peso crítico para o acasalamento" o peso mínimo médio que o rebanho deverá apresentar para obter elevadas taxas de concepção. Assim, a recria das fêmeas deve ser feita em condições nutricionais e sanitárias que permitam atingir esses pesos para que seja possível a ocorrência da primeira concepção aos 22-24 meses ou ainda à menor idade.

Diversos fatores influenciam o peso dos bezerros de corte à desmama: data de parto e idade do bezerro à desmama, estação do ano, idade e raça da vaca e estado nutricional materno no pré e pós-parto (BUTSON et al. 1980). Quanto maior o peso à desmama das bezerras, menor serão as exigências de ganho de peso para que essas atinjam o primeiro acasalamento com desenvolvimento que permita concepção satisfatória (LAMOND, 1970).

É conhecido o efeito do maior desenvolvimento corporal de novilhas sobre o desencadeamento precoce da puberdade (WILTBANK et al. 1969). Existem também evidências de que, para obter elevadas taxas de concepção e manutenção da gestação no primeiro acasalamento, essa fêmea já deverá ter apresentado mais de um ciclo estral antes da estação de cobrição (BYERLEY et al., 1987).

No Estado do Rio Grande do Sul, normalmente, é utilizada a estação reprodutiva para novilhas no período de novembro a janeiro. Assim, para conceber aos 24-26 meses de idade, esta fêmea deverá estar apresentando ciclo estrais regulares a partir de agosto/setembro, fato pouco provável se não forem despendidos maiores cuidados a essa categoria.

O sistema de pastejo "ponta" e "rapador" produz diferentes ofertas de forragem, permitindo ao grupo "ponteiro" realizar maior seletividade sobre a forragem disponível, o que permite melhores desempenhos desse grupo em relação aos "rapadores" (BLASER, 1982). Assim, pode-se obter diferentes respostas segundo a ordem de entrada no potreiro (PEREIRA NETO e LOBATO, 1998).

O objetivo deste trabalho foi avaliar o desenvolvimento de fêmeas bovinas de corte da pós-desmama até o primeiro acasalamento aos 24-26 meses de idade, mantidas em pastejo rotativo "ponta" e "rapador" durante o primeiro e segundo invernos/ primaveras sobre pastagem natural melhorada.

\section{Material e Métodos}

O trabalho foi desenvolvido na Estação Experimental Agronômica (EEA) da Faculdade de Agronomia da Universidade Federal do Rio Grande do Sul, localizada no município de Eldorado do Sul - RS, na região fisiográfica da Depressão Central. Foram utilizadas 76 bezerras cruzas taurinas e zebuínas com variados graus de sangue e mantidas durante o primeiro e segundo invernos/primaveras (ANO-1 e ANO-2, respectivamente) após a desmama em pastagem nativa melhorada de azevém anual (Lolium multiflorum L.) e trevo vesiculoso Yuchi (Trifolium vesiculosum cv Yuchi). Durante o período de verão/ outono, os animais foram alocados em conjunto em potreiro de campo natural da própria EEA.

Ao início do experimento, todos os animais foram pesados e ordenados conforme o peso vivo para, então, serem distribuídos aleatoriamente nos seguintes tratamentos:

Ponta (TP): 38 bezerras, que ingressavam nos potreiros da pastagem melhorada primeiro e consumiam o estrato superior da forragem disponível.

Rapador (TR): 38 bezerras, as quais tinham acesso ao potreiro após a saída do TP e consumiam a forragem remanescente.

O período de utilização pelas bezerras das pastagens melhoradas foi de $108(27 / 07 / 93$ a 12/11/93) e 106 dias (13/07/94 a 27/10/94), no ANO-1 e ANO-2 pós-desmama, respectivamente. Quando não estavam em pastagem melhorada, os animais foram mantidos em grupo único e em potreiro de campo nativo.

O acasalamento foi realizado por meio de monta natural com touros Red Angus (aproximadamente $3 \%$ ). A estação reprodutiva teve duração de 70 dias.

Para a análise dos dados, as bezerras foram agrupadas conforme o peso médio ao início do experimento em Leves (Le) e Pesadas (Pe), de modo que fosse possível avaliar o impacto do sistema de manejo em relação ao peso no momento posterior à desmama.

Os animais foram pesados aproximadamente a cada 28 dias, após jejum total por 16 horas em mangueira.

Foram realizadas avaliações da disponibilidade de forragem a cada mudança de potreiro para determinar a oferta de forragem para cada tratamento.

Os cuidados com manejo sanitário dos animais foi o mesmo empregado ao restante do rebanho bovino da EEA. 
Rev. bras. zootec.

Os dados referentes ao peso vivo (PV) no início e fim dos períodos de pastagem melhorada e o ganho diário médio por período (GDM) foram analisados por intermédio do método GLM do SAS (1988), com número desigual de repetições, sendo empregado o seguinte modelo matemático:

em que

$$
\mathrm{Y}_{\mathrm{ijk}}=\mu+\mathrm{T}_{\mathrm{i}}+\mathrm{GP}_{\mathrm{j}}+\mathrm{T}^{*} \mathrm{GP}_{\mathrm{ij}}+\mathrm{e}_{\mathrm{ijk}}
$$

$\mathrm{Y}_{\mathrm{ijk}}=$ observação referente ao animal $\mathrm{m}$, do tratamento i e grupo de peso j;

$\mu=$ média geral;

$\mathrm{T}_{\mathrm{i}}=$ efeito do tratamento $\mathrm{i}$;

$\mathrm{Gp}_{\mathrm{j}}=$ efeito do grupo de peso $\mathrm{j}$;

$\mathrm{T}^{*} \mathrm{GP}_{\mathrm{ij}}=$ efeito da interação entre o tratamento e o grupo de peso; e

$\mathrm{e}_{\mathrm{ijk}}=$ erro experimental aleatório associado a cada observação.

\section{Resultados e Discussão}

O sistema de pastejo proporcionou peso vivo (PV) significativamente maior para as novilhas do TP em relação ao TR ao final de cada estação de utilização da pastagem nativa melhorada no ANO-1 e ANO-2 (Tabela 1).

A análise dos PV para os grupos de peso inicial demonstraram que as diferenças existentes ao início do experimento foram mantidas até o primeiro acasalamento aos 22-24 meses de idade.

O GDM (Tabela 2) também foi significativamente maior para os animais do TP durante os períodos de utilização da pastagem melhorada, ANO-1 (0,790 vs $0,560 \mathrm{~kg} / \mathrm{dia})$ e ANO-2 (0,753 vs. $0,522 \mathrm{~kg} / \mathrm{dia})$. No decorrer do acasalamento, foi observada significativa inversão no GDM, com o TR apresentando ganho superior ao TP $(0,377$ vs. $0,303 \mathrm{~kg} /$ dia, respectivamente), o que pode ser resultado da maior adaptação dos animais do primeiro tratamento às piores condições da pastagem nativa, quando comparada à pastagem melhorada, ou, ainda, à expressão de ganhos de peso compensatórios, como proposto por RYAN (1990), no decorrer do final da primavera e verão em campo natural.

O sistema de recria deverá estar relacionado com os objetivos produtivos do estabelecimento e a idade em que se pretende realizar o primeiro acasalamento (BAGLEY, 1992). CLANTON (1983) e SMITH et

Tabela 1 - Média dos quadrados mínimos dos pesos vivos das novilhas nas diferentes etapas do experimento

Table 1 - $\quad$ Least square means of live weight of the beef heifers in the different experimental periods

\begin{tabular}{|c|c|c|c|c|}
\hline \multirow[t]{2}{*}{$\begin{array}{l}\text { Período } \\
\text { Period }\end{array}$} & \multicolumn{2}{|c|}{$\begin{array}{l}\text { Tratamento } \\
\text { Treatment }\end{array}$} & \multicolumn{2}{|c|}{$\begin{array}{l}\text { Grupo inicial de peso } \\
\text { Initial liveweight group }\end{array}$} \\
\hline & $\begin{array}{c}\text { Ponta } \\
\text { First grazer }\end{array}$ & $\begin{array}{c}\text { Rapador } \\
\text { Last grazer }\end{array}$ & $\begin{array}{l}\text { Leve } \\
\text { Light }\end{array}$ & $\begin{array}{l}\text { Pesada } \\
\text { Heavy }\end{array}$ \\
\hline $\begin{array}{l}\text { Início-1, kg } \\
\text { Initial-1 }\end{array}$ & 110,3 & 107,6 & 98,8 & $119,2^{*}$ \\
\hline $\begin{array}{l}\text { Fim-1, } \mathrm{kg} \\
\text { End }-1\end{array}$ & $195,6 * *$ & 168,1 & 166,4 & $197,3^{*}$ \\
\hline $\begin{array}{l}\text { Início-2, } \mathrm{kg} \\
\text { Initial-2 }\end{array}$ & $236,4 * *$ & 218,6 & 213,2 & $241,8^{*}$ \\
\hline $\begin{array}{l}\text { Fim-2, } \mathrm{kg} \\
\text { End-2 }\end{array}$ & $316,3 * *$ & 274,1 & 281,5 & $308,8^{*}$ \\
\hline $\begin{array}{l}\text { Início acasalamento, } \mathrm{kg} \\
\text { Mating beginnig }\end{array}$ & $318,5^{* *}$ & 275,7 & 282,0 & $312,2^{*}$ \\
\hline $\begin{array}{l}\text { Fim acasalamento, } \mathrm{kg} \\
\text { Mating end }\end{array}$ & $341,5 * *$ & 304,4 & 307,2 & $338,7^{*}$ \\
\hline $\begin{array}{l}\left({ }^{*}\right) \mathrm{P}<0,05(P<.05) . \\
\left({ }^{* *}\right) \mathrm{P}<0,0001(P<.0001) . \\
\text { Início-1: Peso ao início do } \\
\text { Live weight at beginning of the } \\
\text { Fim-1: Peso no fim do prim } \\
\text { Live weight at the end of the fi } \\
\text { Início-2: Peso ao início do }\end{array}$ & $\begin{array}{l}\text { ro inverno/prim } \\
\text { inter/spring on in } \\
\text { Iverno/primave } \\
\text { ter/spring on imp } \\
\text { do inverno/prin }\end{array}$ & $\begin{array}{l}\text { pastagem m } \\
\text { ative pasture. } \\
\text { stagem melho } \\
\text { ve pasture. } \\
\text { n pastagem } \mathrm{m}\end{array}$ & & \\
\hline $\begin{array}{l}\text { Live weight at beginning of the } \\
\text { Fim-2 Peso no fim do segu } \\
\text { Live weight at the end of the } s \\
\text { Início acasalamento: Peso } \\
\text { Live weight at beginning of the } \\
\text { Fim acasalamento: Peso n } \\
\text { Live weight at the end of the }\end{array}$ & $\begin{array}{l}d \text { winter/spring o } \\
\text { verno/primave } \\
\text { winter/spring on } \\
\text { cio do primeiro } \\
\text { reeding season. } \\
\text { lo primeiro aca } \\
\text { eding season. }\end{array}$ & $\begin{array}{l}d \text { native pasture } \\
\text { stagem melho } \\
\text { native pasture. } \\
\text { nento. } \\
\text { to. }\end{array}$ & & \\
\hline
\end{tabular}


PEREIRA NETO et al.

Tabela 2 - Média dos quadrados mínimos do ganho diário médio (GDM) das novilhas nas diferentes etapas do experimento

Table 2 - Least square means of average daily gain (ADG) of the beef heifers in the differents experimental periods

Período Tratamento Grupo inicial de peso

Period

Treatment

Initial liveweight group

\begin{tabular}{|c|c|c|c|c|}
\hline & $\begin{array}{c}\text { Ponta } \\
\text { First grazer }\end{array}$ & $\begin{array}{c}\text { Rapador } \\
\text { Last grazer }\end{array}$ & $\begin{array}{l}\text { Leve } \\
\text { Light }\end{array}$ & $\begin{array}{l}\text { Pesada } \\
\text { Heavy }\end{array}$ \\
\hline Ano - 1, kg/dia & $0,790 * *$ & 0,560 & 0,626 & 0,723 \\
\hline
\end{tabular}

Year -1

Ano - 2, kg/dia

$0,753 * *$

0,522

0,644

0,632

Year - 2

Estação de acasalamento( $\mathrm{kg} / \mathrm{dia})$

0,303

$0,377^{*}$

0,332

0,349

Breeding season $(\mathrm{kg} /$ day $)$

$\left(^{*}\right) \mathrm{P}<0,05(P<.05)$

$\left({ }^{* *}\right) \mathrm{P}<0,0001(P<.0001)$

al. (1995) concluíram que não existem diferenças sobre o desempenho reprodutivo entre grupos de novilhas que apresentaram ganhos de peso constantes durante a pós-desmama até o primeiro acasalamento, ou quando esses foram concentrados em período de tempo curto, prévio à estação de monta. Aspectos relativos aos custos de cada sistema deverão definir qual a estratégia de ação que poderá ser proposta para cada sistema de produção (PÖTTER et al., 1998).

Analisando o peso adulto médio das vacas de cria do rebanho da EEA, em condição corporal 4 (escala 1-5), observam-se valores entre 450 e $470 \mathrm{~kg}$ PV (PEREIRA NETO, 1996). Se for considerado o proposto por MOSSMANN e HANLY (1977), os quais consideraram a necessidade da fêmea apresentar PV ao início do primeiro acasalamento de aproximadamente $65 \%$ do peso adulto, para obtenção de índices de concepção acima de $85 \%$, as novilhas do presente experimento necessitariam estar com, no mínimo, 292 e $305 \mathrm{~kg}$ PV, objetivo alcançado somente com as novilhas do TP, mas não com as do TR.

A Tabela 3 demonstra, portanto, que, ao serem analisados os grupos de peso em relação ao tratamento imposto durante o ANO-1 e o ANO-2, as fêmeas que exibiram os menores pesos na pósdesmama (Le) e estiveram no tratamento de melhor condição nutricional (TP-Le) apresentaram, ao início do acasalamento, peso médio de $300,7 \mathrm{~kg}$, ou seja, tiveram ganho de peso de $200,8 \mathrm{~kg}$ entre o início do experimento e início do acasalamento. O grupo contemporâneo de peso inicial, que por sua vez foi mantido no grupo "rapador" (TR-Le), com acesso à dieta inferior, apresentaram $263,2 \mathrm{~kg}$ ao início do acasalamento, atingindo o peso desejado para concepção apenas durante a estação reprodutiva. Por outro lado, as fêmeas $\mathrm{Pe}$ apresentaram pesos satisfatórios para a concepção na data de início do acasalamento, a qual evoluiu ainda mais até o final do mesmo. A Figura 1 demonstra graficamente a evolução de cada grupo experimental durante o ANO-1, ANO-2 e primeiro acasalamento.

Dessa forma, pode-se concluir que, caso os animais fossem mantidos em condições não ideais de alimentação, situação semelhante à proporcionada ao grupo TR, apenas os de maior peso à desmama estariam com peso mínimo para obter níveis desejáveis de concepção no primeiro acasalamento. Assim, sendo racionalizados os recursos forrageiros disponíveis, pode-se obter maior contingente de novilhas em condições de conceber na estação reprodutiva.

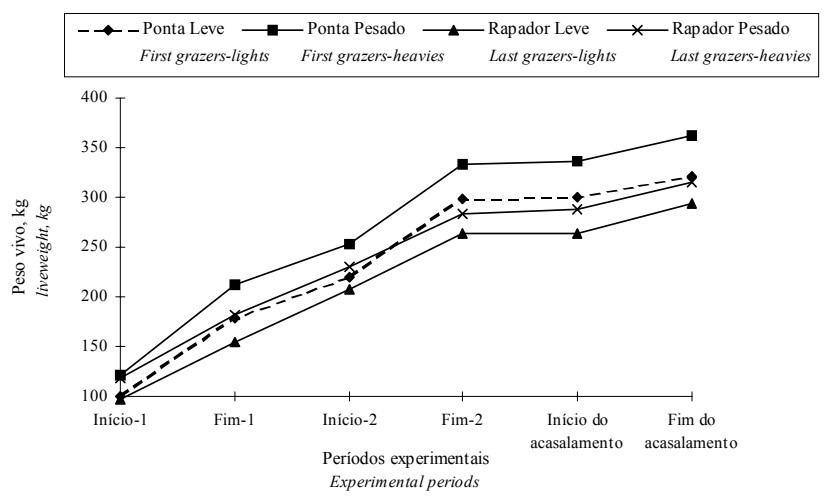

Figura 1 - Efeito da ordem de entrada na pastagem sobre a evolução do peso vivo de cada grupo de peso inicial, conforme as distintas etapas experimentais.

Figure 1 - Effect of grazing order on liveweight evolution for initial bodyweight group, in each experimental period. 
Tabela 3 - Média dos quadrados mínimos para os pesos médios dos diferentes tratamentos e grupos de peso inicial, em cada etapa do experimento

Table 3 - Least square means for live weights of different treatments and initial live weight group, in each experimental period

\begin{tabular}{|c|c|c|c|c|}
\hline \multirow[t]{2}{*}{$\begin{array}{l}\text { Período } \\
\text { Period }\end{array}$} & \multicolumn{2}{|c|}{$\begin{array}{l}\text { Ponta }(\mathrm{TP}) \\
\text { First grazers }\end{array}$} & \multicolumn{2}{|c|}{$\begin{array}{l}\text { Rapador (TR) } \\
\text { Last grazers }\end{array}$} \\
\hline & $\mathrm{Le}$ & $\mathrm{Pe}$ & $\mathrm{Le}$ & $\mathrm{Pe}$ \\
\hline Início-1 & $99,9^{\mathrm{B}}$ & $120,7^{\mathrm{A}}$ & $97,6^{\mathrm{B}}$ & $117,5^{\mathrm{A}}$ \\
\hline Initial-1 & & & & \\
\hline Fim-1 & $178,4^{\mathrm{B}}$ & $212,8^{\mathrm{A}}$ & $154,4^{\mathrm{C}}$ & $181,7^{\mathrm{B}}$ \\
\hline $\begin{array}{l}\text { End-1 } \\
\text { Início-2 }\end{array}$ & $219,2^{\mathrm{C}}$ & $253,5^{\mathrm{A}}$ & $207,3^{\mathrm{D}}$ & $230,0^{\mathrm{B}}$ \\
\hline $\begin{array}{l}\text { Initial-2 } \\
\text { Fim-2 } \\
\text { End-2 }\end{array}$ & $298,9^{\mathrm{B}}$ & $333,6^{\mathrm{A}}$ & $264,1^{\mathrm{C}}$ & $284,1^{\mathrm{C}}$ \\
\hline $\begin{array}{l}\text { End-2 } \\
\text { Início do acasalamento } \\
\text { Mating heoinning }\end{array}$ & $300,7^{\mathrm{B}}$ & $336,3^{A}$ & $263,2^{\mathrm{D}}$ & $288,2^{\mathrm{C}}$ \\
\hline $\begin{array}{l}\text { Fim do acasalamento } \\
\text { Mating end }\end{array}$ & $321,1^{\mathrm{B}}$ & $361,9^{\mathrm{A}}$ & $293,3^{C}$ & $315,5^{\mathrm{B}}$ \\
\hline
\end{tabular}

$A B$ - Médias seguidas pela mesma letra, na mesma linha, não diferem.

$A B$ - Means followed by the same letter, in the same row, do not differ.

Le: Leves (Lights); Pe: Pesadas (Heavies).

Início-1: Peso ao início do primeiro inverno/primavera em pastagem melhorada.

Live weight at beginning of the first winter/spring on improved native pasture.

Fim-1: Peso no fim do primeiro inverno/primavera em pastagem melhorada.

Live weight at the end of the first winter/spring on improved native pasture.

Início-2: Peso ao início do segundo inverno/primavera em pastagem melhorada.

Live weight at beginning of the second winter/spring on improved native pasture.

Fim-2 Peso no fim do segundo inverno/primavera em pastagem melhorada.

Live weight at the end of the second winter/spring on improved native pasture.

Início do acasalamento: Peso ao início do primeiro acasalamento.

Live weight at beginning of the first breeding.

Fim do acasalamento: Peso no fim do primeiro acasalamento.

Live weight at the end of the first breeding season.

Os resultados demonstram que, quando se desejam melhores condições de recria para novilhas de reposição, com correto programa de manejo nutricional e sanitário, mesmo havendo diferenças de peso na desmama, pode-se obter desenvolvimento satisfatório até o início do primeiro acasalamento, principalmente se houver maior cuidado com as bezerras de menor peso na pós desmama. Resultados semelhantes foram encontrados com outros grupos de animais por BERETTA e LOBATO (1996) e PEREIRA e LOBATO (1998).

Ficam, portanto, evidenciadas as necessidades de se implementarem práticas de manejo que permitam alavancar o desenvolvimento das novilhas de reposição, permitindo que ingressem precocemente no rebanho de cria.

\section{Conclusões}

Os sistemas de pastejo "ponta" e "rapador" promoveram maior ganho de peso durante o primeiro e segundo invernos/primaveras, para o grupo ponteiro.

Bezerras leves em relação à média grupal, se dispostas em grupo que privilegie o ganho de peso, apresentam desenvolvimento e boas condições de serem acasaladas aos dois anos de idade, com $65 \%$ do peso vivo do rebanho adulto de origem.

Sistemas de manejo que permitam privilegiar um grupo animal em relação a outro devem ser implementados para corrigir diferenças de peso entre animais, resultando em maior homogeneidade entre os grupos ao final do período e permitindo maior número de novilhas com peso satisfatório para a concepção.

\section{Referências Bibliográficas}

BAGLEY, C.P. 1992. Nutritional management of replacement heifers: a review. J. Anim. Sci., 71(11):3155-3163.

BERETTA, V., LOBATO, J.F.P. 1996. Efeitos da ordem de utilização de pastagens melhoradas no ganho de peso e desempenho reprodutivo de novilhas de corte. $R$. Soc. Bras. Zootec., 25(6):1196-1206.

BLASER, R.E. 1982. Integrated pasture and animal management. Trop. Grass., 16(1):9-23.

BOLZE, R., CORAH, L.R. 1993. Selection and development of replacements heifers. Manhattan: Cooperative Extension Service, Kansas City University. 9 p.

BUTSON, S., BERG,R.T., HARDIN, R.T. 1980. Factors influencing weaning weights of range beef and dairy beef calves. Can. J. Anim. Sci., 60(3):727-742.

BYERLEY, D.J., STAIGMILER, R.B., BERARDINELLI, J.G. 
1987. et al. Pregnancy rate of beef heifers bred either on puberal or third estrus. J. Anim. Sci., 65(3):645-650.

CLANTON, D.C., JONES, L.E., ENGLAND, M.E. 1983. Effects of rate and time of gain after weaning on the development of replacements beef heifers. J. Anim. Sci., 56(2):280-285.

GRAWUNDER, A.F., MIELITZ NETO, C.G.A. 1979. Pecuária de corte no Sul do Brasil: Que caminhos tomar? R. Econ. Rural. 17(4):119-136.

HOLMES, P.R. 1989. The opportunity of lifetime. Reproductive efficiency in beef cattle herd. New Jersey: MSD-AgVet, Merck. 54 p.

LAMOND, D.R. 1970. The influence of undernutrition on reproduction in the cow. Anim. Breed. Abst., 38(3):359-372.

MOSSMAN, D.H., HANLY, G.J. 1977. A theory of beef production. N. Z. Vet. J., 25(1):96-100.

PARSONS, S.D., ALLISON, C.D. 1991. Grazing management as it affects nutrition, animal production and economics of beef production. Vet. Clin. North America: Food Practice, 7(1):77-94.

PEREIRA NETO, O.A. Efeito da ordem de utilização de pastagens nativas melhoradas no desenvolvimento e comportamento reprodutivo de novilhas de corte. Porto Alegre, 1996. 143 f. Dissertação (Mestrado em Zootecnia) - Programa de Pós-Graduação em Agronomia. Universidade Federal do Rio Grande do Sul, 1996.

PEREIRA NETO, O.A., LOBATO, J.F.P. 1998. Efeitos da ordem de utilização de pastagens nativas melhoradas no desenvolvimento e comportamento reprodutivo de novilhas de corte. R. Bras. Zootec., 27(1):60-65.
PÖTTER, L., LOBATO, J.F.P., MIELITZ NETO, C.G.A. 1998. Produtividade de um modelo de produção para novilhas de corte primíparas aos dois, três ou quatro anos de idade. $R$. Bras. Zootec., 27(3):613-619.

RYAN, W.J. 1990. Compensatory growth in cattle and sheep. Nut. Abst. Rev. (Serie B), 60(9):653-664.

SAS - Statistical Analisys System. Language guide for personal computer, 6.03, Cary: SAS Institute, 1988, v. 12, 378 p.

SMITH, J.M., LAMB, G.C., MINTON, J.E. et al. 1995. Influence of timing of gain on reproductive performance in beef heifers. J. Anim. Sci., v. 73, suppl. 1, p. 233.

WILTBANK, J.N., KASSON, C.W., INGALS, J.E. 1969. Puberty in crossbred and straightbred beef heifers on two levels of feed. J. Anim. Sci., 29(4):602-605.

Recebido em: $12 / 02 / 98$

Aceito em: 27/08/98 